\title{
MECHANISMS OF THE EARLY FATIGUE DAMAGE IN METALLIC MATERIALS
}

The early stages of the fatigue damage in f.c.c. and b.c.c. metals are studied using high resolution techniques. The localization of the cyclic plastic strain results in formation persistent slip bands (PSBS) with specific dislocation structure. Characteristic surface relief is formed at locations where PSBs emerge on the surface. It consists of extrusions and intrusions separated by the original flat surface. Atomic force microscopy is used to study the details of the surface relief. The experimental findings are discussed in terms of the point defect model of fatigue crack initiation.

\section{Introduction}

The basic facts concerning the surface relief formation in fatigued metals were documented already at the beginning of the $20^{\text {th }}$ century by Ewing and Humfrey [1] by observing the surface of fatigued Swedish iron. The optical microscope used in these and later studies could not reveal details of the emerging extrusions and intrusions and eventually the early initiation of fatigue cracks. Later studies using replicas in transmission electron microscope or classical scanning electron microscope (see. e.g. [2]) yield higher resolution but it is not possible to obtain quantitative information in three dimensions.

Transmission electron microscopy allowed studying dislocation structure of fatigued metals [2]. Early studies in single crystals revealed specific structure of the bands, responsible for the localization of the cyclic strain - persistent slip bands (PSBs). Oriented foil technique [3] allows studying dislocation structure in individual grains of polycrystalline materials. PSBs accommodate high cyclic plastic strain. Due to high cyclic plastic strain amplitude localized in PSBs pronounced surface relief in the form persistent slip markings (PSMs) is formed in areas where PSBs emerge on the surface.

In this contribution the characteristic dislocation structures corresponding to cyclic slip localization in polycrystalline f.c.c. and b.c.c. stainless steels observed using transmission electron microscope are shown. The characteristic surface relief produced during cyclic straining of these materials - PSMs - using AFM (Topometrics) is documented. The experimental data are used to discuss the models of fatigue crack initiation.

\section{Experimental}

Polycrystalline specimens made of $316 \mathrm{~L}$ austenitic stainless steel and $\mathrm{X} 10 \mathrm{CrAl} 24$ ferritic stainless steel were used in the present study. Smooth cylindrical specimens having $8 \mathrm{~mm}$ in diameter and $12 \mathrm{~mm}$ in gage length were slightly notched to produce surface suitable for surface observations. The dimensions of the specimen are shown in Fig. 1. The area of the notch was mechanically and electrolytically polished to facilitate the observations of the surface relief. The specimens were cycled in computer controlled MTS 880 electrohydraulic machine. Symmetrical strain cycle with the strain rate $\dot{\epsilon}=2.5 \times 10^{-3} \mathrm{~s}^{-1}$ was applied at room temperature. The plastic strain amplitude derived from the half-width of the hysteresis loop was kept constant using the computer "outer loop". Further experimental details concerning the materials and testing procedure can be found elsewhere [3, 4].
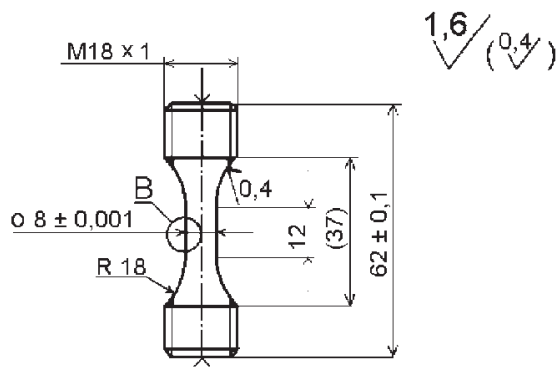

$B(\mathrm{M} \mathrm{10:1)}$
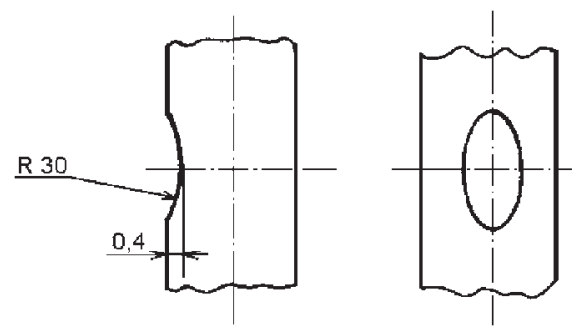

Fig. 1 Dimensions of the specimen with a shallow notch

\footnotetext{
* Jaroslav Polák, Jiří Man, Martin Petrenec

Institute of Physics of Materials, Academy of Sciences of the Czech Republic, Žižkova 22, Brno, Czech Republic, polak@ipm.cz
} 
The cycling was interrupted at a selected number of cycles; the specimen was taken from the testing machine and inspected in AFM. Replica technique was applied to reveal the depth and the shape of extrusions and intrusions [5].

Some fatigued specimens were cut and thin foils with the foil plane parallel to the specimen axis were produced. They were observed in transmission electron microscope Philips CM12. Orientation of the foil relative to the loading axis was determined with the help of electron diffraction and Kikuchi lines.

\section{Results}

\subsection{Dislocation structures}

Dislocation structures corresponding to cyclic slip localization were studied mostly in polycrystalline specimens cycled with medium and low plastic strain amplitudes. Previous studies in austenitic steel and in austenitic grains of duplex steel [6-8] revealed the presence of specific bands (PSBs) with irregular ladder structure embedded in the matrix. The density of individual PSBs is highest for medium plastic strain amplitudes $\left(\sim 2 \times 10^{-3}\right)$.

Figure 2 shows characteristic dislocation structures in austenitic and in ferritic stainless steels in specimens cycled with medium plastic strain amplitudes up to the end of the fatigue life. Orientation of individual grains is shown by the position of the stress axis in a stereographic triangle. The trace of the primary slip plane and Schmid factors of mostly stressed systems are also shown. Individual ladder-like bands, parallel to the primary slip plane in Fig. 2 a consist of alternating dislocation-rich walls and dislocation-poor channels, both approximately perpendicular to the foil plane. This structure corresponds to the dislocation arrangement in fatigued single crystals [2].

Ladder-like dislocation structures were also found in fatigued ferritic stainless steel cycled with constant low plastic strain amplitude. Figure $2 b$ shows a micrograph of a grain oriented for single slip in a section close to (12 1$)$ plane with three bands of ladderlike structure parallel to the traces of $(\overline{101})$ or $(\overline{112})$ planes. The matrix is formed by random arrangement of dislocations [9]. Dislocation walls consisting of dipole and multipole configurations are more regular than in case of austenitic steel (Fig. 2a). Screw dislocation segments in the channels are hanging between neighbor dislocation walls.

\subsection{Surface relief evolution}

Typical shapes of PSMs in 316L steel fatigued to fracture as recorded by AFM are shown in Fig. 3. Since the real shape of PSMs is distorted in the AFM [5] it was necessary to record not only the AFM image of the metallic surface (Fig. 3a) but also the inverted AFM image of the plastic replica taken at the identical location (Fig. 3b). Comparing these two images enough information on the real shape of typical PSMs in fatigued $316 \mathrm{~L}$ steel can be obtained. The band-like extrusions emanate from the material in a direction of the active Burgers vector and their height fluctuates only moderately. One or two thin parallel intrusions, observed on the image of plastic replica (Fig. 3b), penetrate into the material at the boundary between PSB and the matrix, preferably on the side of the extrusion where the angle between the surface and the slip plane emerging from the crystal is acute.
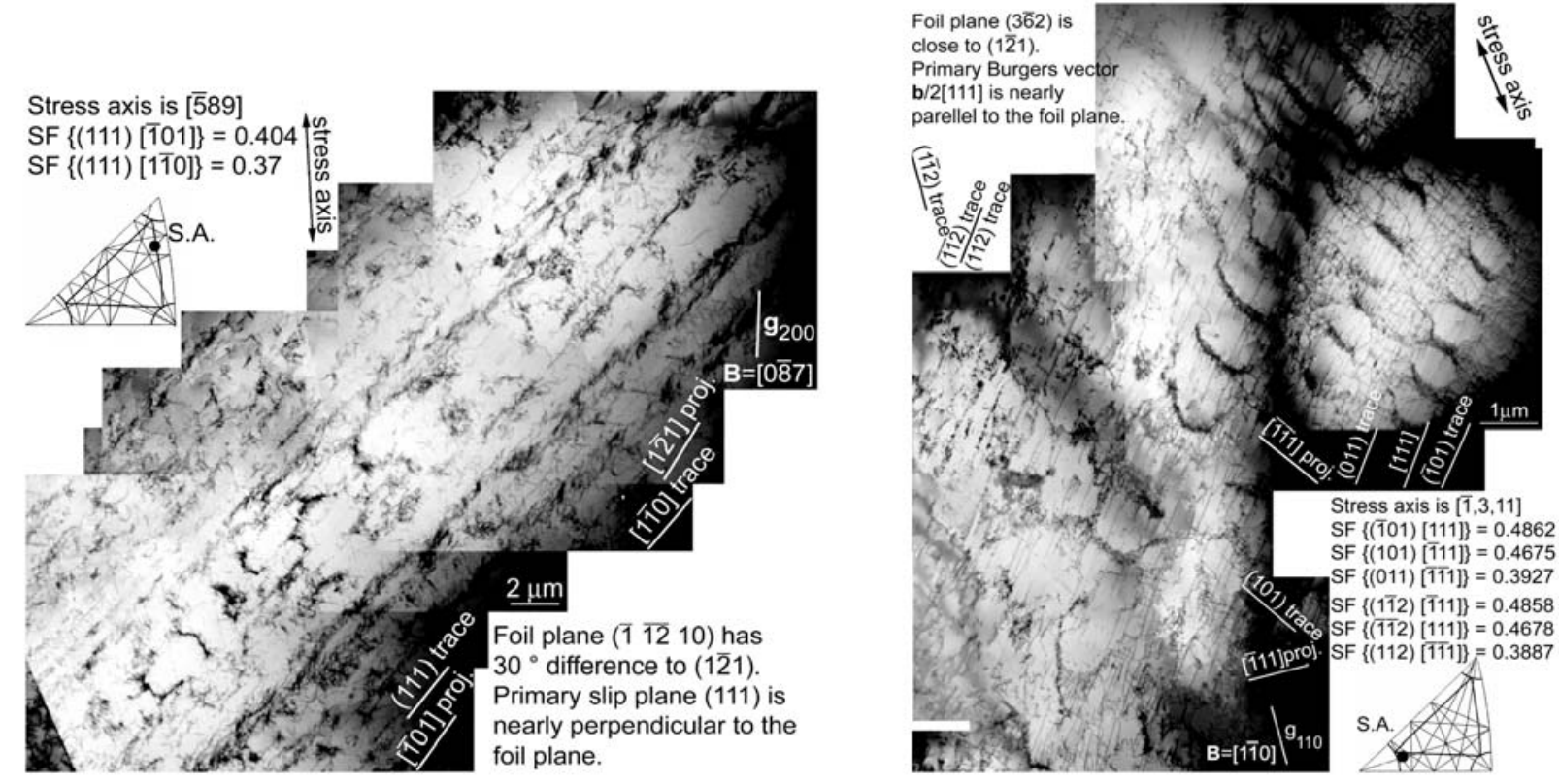

Fig. 2 Ladder-like structures of PSBs and matrix in specimens cycled to fracture (a) austenitic steel, $\epsilon_{a p}=1 \times 10^{-3}$ (b) ferritic steel, $\epsilon_{a p}=2 \times 10^{-3}$. (Direction of the stress axis (S.A.) is shown in a basic triangle). 

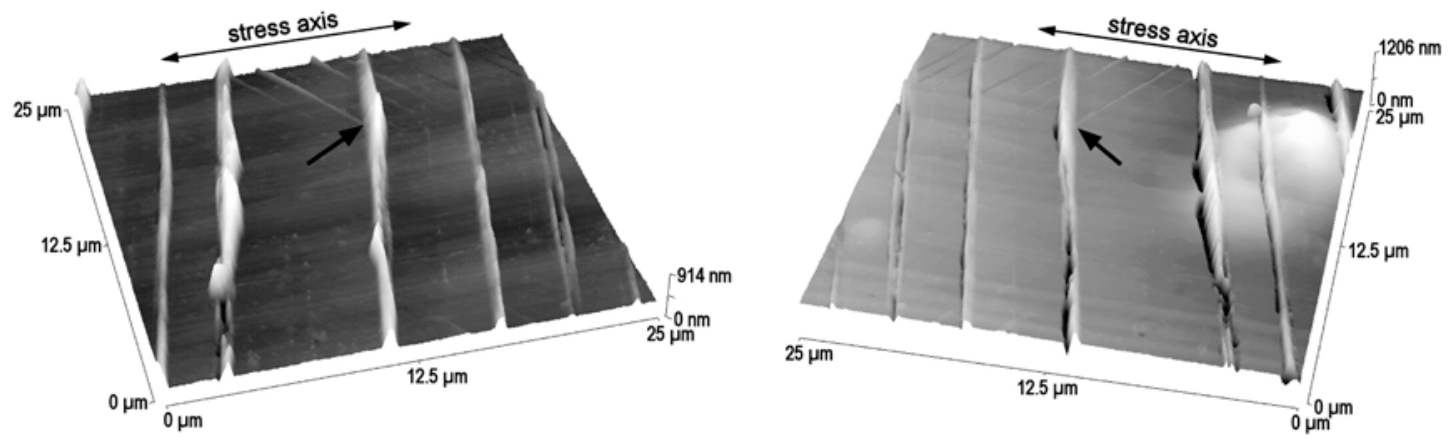

Fig. 3 AFM images of the surface relief of individual PSMs in $316 \mathrm{~L}$ steel on the metallic specimen (a) and on the plastic replica (b); $\epsilon_{a p}=2 \times 10^{-3}, N=20000$ cycles.

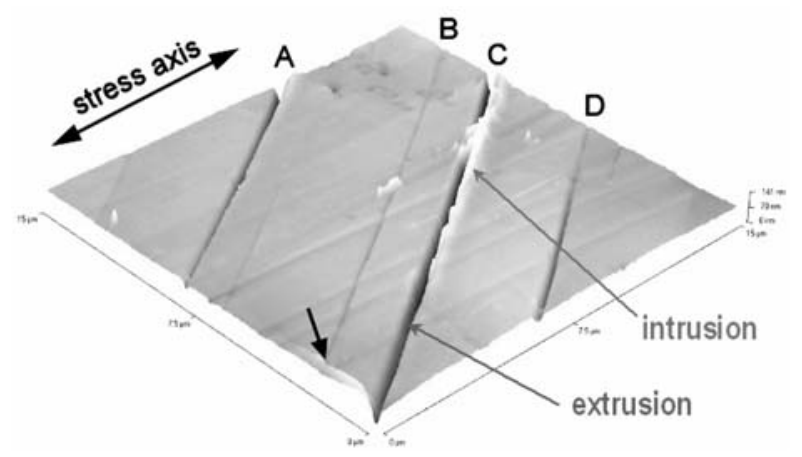

Fig. 4 AFM image of the plastic replica of the austenitic grain after 500 loading cycles with $\epsilon_{a p}=1 \times 10^{-3}$.

The growth of extrusions and intrusions in constant plastic strain amplitude cycling has been followed quantitatively using AFM. The extrusions start to grow very early in life $(0.1 \% \mathrm{Nf})$. The initial rate of extrusion growth is high, decreases later and for most

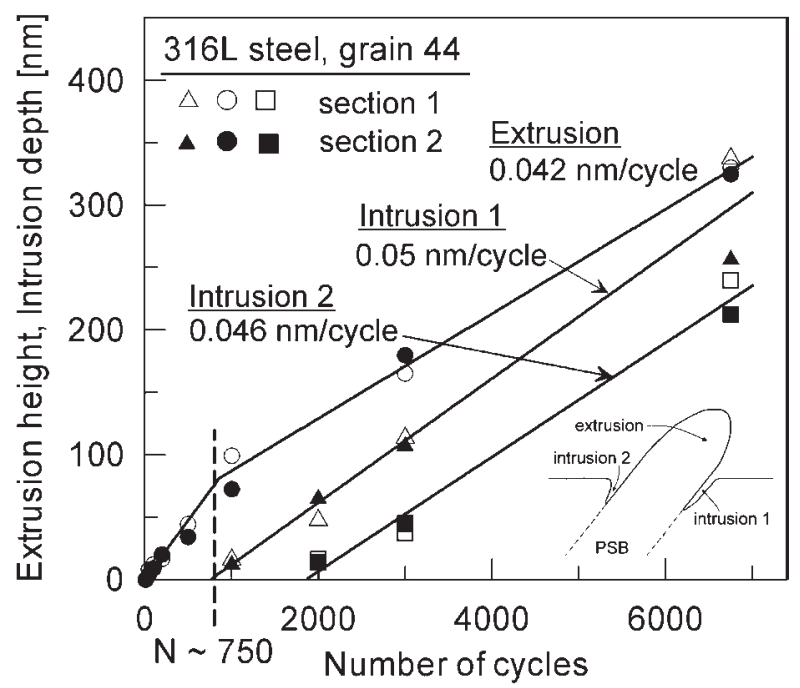

Fig. 5 Simultaneous growth of extrusions and intrusions in the grain Oof austenitic steel at the beginning of cycling with $\epsilon_{a p}=1 \times 10^{-3}$. of the fatigue life the stabilized value $2.5 \times 10^{-11} \mathrm{~m} /$ cycle in cycling with constant plastic strain amplitude $2 \times 10^{-3}$ is reached [10]. The growth of intrusions can be measured using plastic replica technique. Only early stages of intrusion formation could be documented since intrusions are very thin and deep and plastic replica reproduces their shape only when their depth is less than $0.5 \mu \mathrm{m}$, exceptionally up to $1 \mu \mathrm{m}$. Figure 4 shows the AFM image of the plastic replica of a grain of $316 \mathrm{~L}$ steel cycled with plastic strain amplitude $\epsilon_{a p}=1 \times 10^{-3}$ for 500 cycles $\left(1.1 \% N_{f}\right)$. Four PSMs denoted A, B, C, D are present. An intrusion starts growing at one side of an extrusion $\mathrm{C}$ while extrusions in all PSMs are already well developed. The first signs of this intrusion were detected at 350 cycles $\left(0.8 \% N_{f}\right)$. Figure 5 shows the height of an extrusion and the depth of parallel intrusions accompanying the extrusion early in fatigue life. The plot and also more detailed observations [10] reveal that while the extrusions start very early in fatigue life the intrusions appear and start to grow after some lag $(\sim 350$ cycles in this grain). In most cases the rate of intrusion growth is much higher than that of the extrusion. This could be connected with the fact that intrusions are much thinner than extrusions.

Similar observations on the shape and the growth of extrusions and intrusions were made on ferritic stainless steel. Figure 6 shows the AFM image of one grain of ferritic stainless steel cycled with $\epsilon_{a p}=2 \times 10^{-3}$ for 9000 cycles (close to $N_{f}$ ). Figure 6a shows the image of the metallic surface and Fig. $6 \mathrm{~b}$ the image of the plastic replica. While only extrusions seem to be detected in Fig. 6a, Fig. $6 \mathrm{~b}$ shows both extrusions and parallel intrusions. The variation of the extrusion height and intrusion depth along PSMs is higher than in austenitic steel. Therefore for the quantitative measurement of extrusion growth in ferritic stainless steel the mean integral height of extrusions was evaluated [11]. Also in ferritic steel the initial growth rate of extrusions is higher and stabilizes later for most of the fatigue life.

\section{Discussion}

Experimental data on the dislocation structure of PSBs and on the details of the surface relief simultaneously with the knowledge of the dislocation motion and interactions in metallic materials allow us to specify the mechanisms leading to the early formation of 


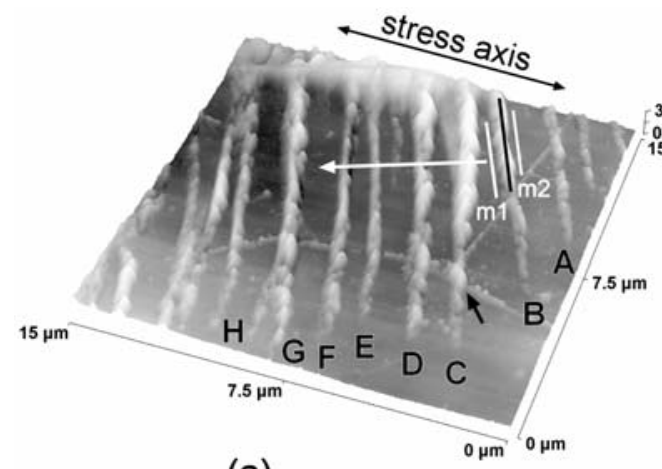

(a)

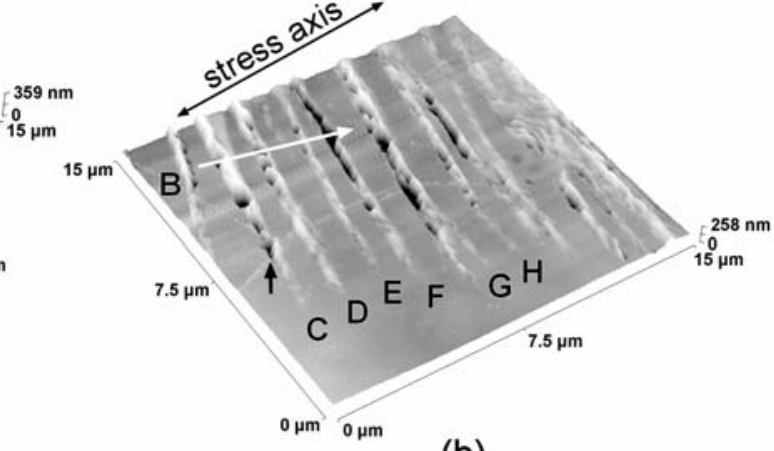

(b)

Fig. 6 AFM images showing the surface relief of individual PSMs in a grain of ferritic steel taken on the metallic specimen (a) and on the plastic replica (b); $\epsilon_{a p}=2 \times 10^{-3}, N=9000$.

the surface damage in the form of PSMs. Fig. 7 shows schematically the dislocation arrangement of a PSB. It consists of alternat-

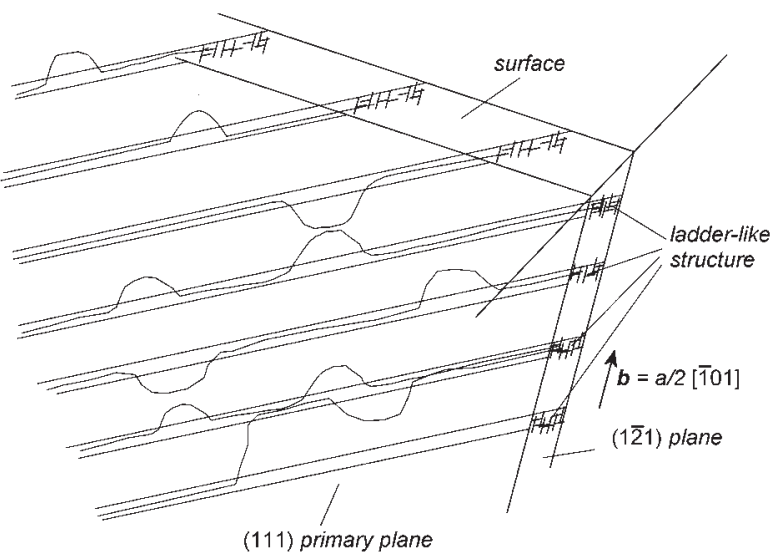

Fig. 7 Schematic picture showing dislocation structure of a PSB.

ing dislocation walls composed from edge dipoles and multipoles alternating with dislocation free channels. Dislocation loops are emitted from the walls and expand in the primary slip plane without obstruction into the neighbor channel. When reaching the opposite wall the edge segment is built in the neighbor wall in the multipole configuration. The screw parts move apart carrying the majority of the local plastic strain until they annihilate by cross slip with a screw dislocation segment on a parallel plane moving in the opposite direction. This deformation mechanism can result only in random relief formation. To account for the extrusion and intrusion formation some redistribution of matter must be considered.

Point defect models of fatigue crack initiation predict the formation of extrusions and/or intrusions. Formation of point defects in cyclic straining has been proved experimentally [12]. Essmann et al. [13] (EGM model) consider the formation of unit dipoles, i.e. rows of vacancies or interstitials by encountering two opposite edge dislocations on a neighbor planes or by the motion of a nonconservative jog on a screw dislocation. Due to lower formation energy vacancy production prevails. Only a static extrusion whose height is proportional to the saturated vacancy concentration was considered in EGM model [13]. Polák's model [14] extends the EGM model by considering the migration of vacancies from the PSB to the matrix. The reversed migration of the atoms from the matrix to the PSB leads to an accumulation of matter in a PSB. The compression stresses are relaxed by plastic straining and result in a steady growth of an extrusion in agreement with experiment. In the areas, where vacancies are annihilated in the matrix, i.e. mostly at the boundary between the matrix and PSB, the matter is missing and intrusions arise and steadily deepen.

The vacancy production rate $p\left(\epsilon_{a p}\right)$ depends on the local plastic strain amplitude $\epsilon_{a p}$. The net vacancy production rate is reduced by two processes: (i) the sweeping and annihilation of vacancies by moving dislocation loops (ii) the migration of vacancies from the PSB to the matrix i.e.

$$
\frac{d c}{d N}=p\left(\epsilon_{a p}\right)-(A+M(T)) c
$$

where $A$ is the relative annihilation rate of vacancies (fraction of vacancy concentration annihilated in one cycle) mostly due to encounter with moving edge dislocations and $M(T)$ is the vacancy migration rate characterizing the flow of vacancies out of the PSB to the matrix (fraction of vacancy concentration leaving the $\mathrm{PSB} /$ matrix boundary). The relative annihilation rate $A$ is independent of temperature but the vacancy migration rate $M(T)$ is strongly temperature dependent and is determined by the vacancy diffusion coefficient and geometrical parameters, e.g. thickness of the PSB.

Vacancy concentration will be obtained by integrating eq. (1)

$$
c=\frac{P}{A+M(T)}[1-\exp (-(A+M(T)) N]
$$

Saturated vacancy concentration $c_{S}=p /(A+M(T))$ in PSB will be achieved for each temperature. The flow of atoms from the matrix to the PSB is equal to the net flow of vacancies from PSB to the matrix in saturation. The steady growth rate of the extrusion height $d h / d N$ is thus 


$$
\frac{d h}{d N}=l\left(\frac{d c}{d N}\right)_{P S B-\text { matrix }}=l \frac{p M(T)}{A+M(T))}
$$

where $l$ is the depth of the PSB bellow the surface. The growth rate of the extrusion is proportional to the vacancy production rate and depends on the fraction of vacancies annihilated athermally and by migration to the matrix. Migration rate is proportional to the jump frequency and is given by the term $\exp \left(-E_{M} / k T\right)$ where $\mathrm{k}$ is Boltzmann constant and $E_{M}$ vacancy migration energy. The shape and the depth of intrusions depend on the arrangement of edge dislocations in the matrix.

The production of vacancies in PSB and their migration to the matrix has been modeled in two dimensions using the Monte Carlo method [15]. The growth of extra-vacancy concentration has been obtained. The width of a growing extrusion is the same as the width of a PSB while two thin and deep intrusions are produced at the boundary between the PSB and the matrix. After the initial delay, corresponding to the formation of saturated vacancy concentration, a linear growth of extrusion and two parallel intrusions is observed. Intrusion represents a very sharp notch and irreversible cyclic straining leads to the formation of new surfaces and thus to crack initiation.

\section{Conclusions}

The analysis of experimental results on the early fatigue damage in polycrystalline f.cc. and b.c.c. steels and the comparison with physically based models lead to the following conclusions:

(i) Localization of the cyclic plastic strain results in the early formation of PSBs.

(ii) PSBs have specific dislocation structure consisting of alternating thin dislocation rich walls and wide dislocation free channels.

(iii) Intensive cyclic plastic straining within the PSB leads to the dislocation interaction and vacancy formation and finally PSMs consisting of an extrusion and parallel intrusions arise on the surface.

(iv) Surface intrusions represent high stress and strain raisers and cyclic plastic strain within PSB is concentrated to the tip of the intrusions and usually one intrusion changes into a crack capable of further growth.

\section{Acknowledgements}

The authors acknowledge the support by the grant No. 1QS200410502 of the Academy of Sciences of the Czech Republic.

\section{References}

[1] EWING, J. A., W. HUMFreY, J. C.: Phil. Trans. Roy. Soc. A200 (1903) 241-250.

[2] POLÁK, J.: Comprehensive Structural Integrity, I. Milne, R. O. Ritchie, B. Karihaloo, (Eds.) Vol. 4, Elsevier, 2003, Amsterdam, pp. 1-39.

[3] POLÁK, J., PETRENEC, M., MAN, J.: Mater. Sci. Eng. A400-401 (2005) 405-408.

[4] POLÁK, J., OBRTLÍK, K., HÁJEK, M.: Fatigue Fract. Eng. Mater. Struct. 17 (1994) 773-782.

[5] POLÁK, J., MAN, J., OBRTLÍK, K., KRUML, T.: Metallkd. 94 (2003) 1327-1330.

[6] GERLAND, M., MENDEZ, J., VIOLAN, P., AIT SAADI, B.: Mater. Sci. Eng. A118 (1989) 83-95.

[7] OBRTLÍK, K., KRUML, T., POLÁK, J.: Mater. Sci. Eng. A187 (1994) 1-9.

[8] KRUML, T., POLÁK, J., OBRTLÍK, K., DEGALLAIX, S. : Acta Mater. 45 (1997) 5145-5151.

[9] PETRENEC, M., POLÁK, J., OBRTLÍK, K., MAN, J.: Acta Mater.54 (2006) 3429--3443.

[10] MAN, J., OBRTLÍK, K., POLÁK, J.: Mater. Sci. Eng. A351 (2003) 123-132.

[11] MAN, J., PETRENEC, M., OBRTLÍK, K., POLÁK, J.: Acta Mater. 52 (2004) 5551-5561.

[12] POLÁK, J.: Mater. Sci. Eng. 89.(1987) 35-43.

[13] ESSMANN, U., GÖSELE, U., MUGHRABI, H.: Phil. Mag. A 44 (1981) 405-426.

[14] POLÁK, J.: Mater. Sci. Eng. 92 (1987) 71-80.

[15] POLÁK, J., ZOUHAR, L. to be published. 